\title{
Myeloid/Lymphoid Neoplasms with PDGFRA Rearrangement
}

National Cancer Institute

\section{Source}

National Cancer Institute. Myeloid/Lymphoid Neoplasms with PDGFRA Rearrangement.

NCl Thesaurus. Code C84275.

Hematologic neoplasms characterized by the rearrangement of the PDGFRA gene, most often resulting in the formation of FIP1L1-PDGFRA fusion transcripts. Patients usually present with chronic eosinophilic leukemia, and less often with acute myeloid leukemia or T-lymphoblastic leukemia. 